\title{
Đánh giá khả năng dự báo mưa do bão bằng mô hình RAMS
}

\author{
Công Thanh ${ }^{1, *}$, Trần Tiến Đạt ${ }^{2}$, Vũ Thanh Hằng ${ }^{1}$ \\ ${ }^{1}$ Truò̀ng Đại học Khoa học Tư Nhiên, ĐHQGHN, 334 Nguyễn Trãi, Hà Nội, Việt Nam \\ ${ }^{2}$ Trung tâm ứng dụng công nghệ và bồi duõng nghiệp vu KTTV\&MT, TTKTTVQG \\ Nhận ngày 08 tháng 8 năm 2016 \\ Chỉnh sửa ngày 26 tháng 8 năm 2016; Chấp nhận đăng ngày 16 tháng 12 năm 2016
}

\begin{abstract}
Tóm tắt: Trong nghiên cứu này nhóm tác giả đánh giá khả năng dự báo mưa do bão bằng mô hình RAMS với thời hạn 3 ngày cho khu vực Việt Nam có sử dụng hai sơ đồ đối lưu Kuo và Kainfritsch. Nhóm nghiên cứu tiến hành các phương án thử nghiệm bằng phương pháp lưới lồng và thay đổi luân phiên hai sơ đồ đối lưu này làm lưới 1 và lưới 2 , từ đó đưa đến kết quả: Với hạn dự báo $24 \mathrm{~h}$ nên sử dụng phương án $\mathrm{K}-\mathrm{K}$ để dự báo ngưỡng mưa vừa $(16-50 \mathrm{~mm})$ và mưa to $(50-$ $100 \mathrm{~mm}$ ); Hạn dự báo $48 \mathrm{~h}$ nên sử dụng phương án K-KF để dự báo mưa do bão cho ngưỡng mưa vừa và phương án $\mathrm{K}-\mathrm{K}$ để dự báo cho ngưỡng mưa to; Hạn dự báo $72 \mathrm{~h}$ nên sử dụng phương án $\mathrm{K}$ $\mathrm{KF}$ để dự báo ngưỡng mưa vừa và mưa to. Ngưỡng mưa trên $100 \mathrm{~mm}$, các hạn dự báo cho kết quả dự báo kém, hầu như không dự báo được. Từ những kết quả trong nghiên cứu này có thể giúp dự báo viên có thêm thông tin phục vụ cho việc dự báo mưa do bão trên khu vực Việt Nam.
\end{abstract}

Tù khóa: Nghiên cứu và dự báo mưa, bão.

\section{Mở đầu}

Bão là một trong những hiện tượng thời tiết vô cùng nguy hiểm, gây ảnh hưởng lớn tới nền kinh tế, xã hội của nhiều quốc gia ven biển, đặc biệt là đối với những quốc gia có đường bờ biển dài như Việt Nam. Khi nói tới bão người ta thường đề cập tới những hệ quả mà nó gây ra như gió mạnh, sóng lớn, nước dâng do bão và đặc biệt mưa lớn. Theo một nghiên cứu của Prat [1], khi dựa vào số liệu toàn cầu của NOAA, số liệu vệ tinh, số liệu phân tích và đưa ra kết luận mưa do bão ảnh hưởng tới một diện tích khá lớn trên hầu hết các châu lục, cụ thể khu vực Đông Á, bão nhiệt đới cung cấp lượng

\footnotetext{
*Tác giả liên hệ. ĐT.: 84-946180348

Email: thanhc@vnu.edu.vn
}

mưa khoảng $11 \%$ lượng mưa hằng năm của khu vực (chỉ đứng sau đông Phi 12\%). Với ảnh hưởng lớn như vậy nên những nghiên cứu về mưa trong bão ở khu vực này và đặc biệt là ở Việt Nam là một vấn đề rất cấp thiết.

Hiện nay có rất nhiều công trình nghiên cứu dự báo mưa do ảnh hưởng của bão trên thế giới như công trình của Chen L. và ccs (2010) [2] đã tiến hành mô tả vùng mưa có liên quan đến bão đổ bộ và những hệ thống thời tiết tương ứng, đồng thời tác giả cũng chỉ ra những cơ chế vật lý quan trọng ảnh hưởng tới lượng mưa và phân bố mưa do bão đổ bộ. Nhóm nghiên cứu đưa ra các cơ chế ảnh hưởng đến lượng mưa do bão gồm: nguồn ẩm, vận chuyển ngoại nhiệt đới, địa hình, hệ thống đối lưu qui mô vừa, lớp biên. Từ việc nghiên cứu thử nghiệm, nhóm nghiên cứu đưa ra kết luận, nguồn ẩm trong lớp 
mực thấp là nguồn cung cấp mưa khi bão đổ bộ, hoặc khi bão đổ bộ tương tác với sự khởi phát gió mùa, cũng như vận chuyển thẳng đứng của nguồn hơi nước trong đất liền (hồ, sông, hơi nước bão hòa gần bề mặt...) góp phần làm tăng đáng kể lượng mưa của bão đổ bộ.

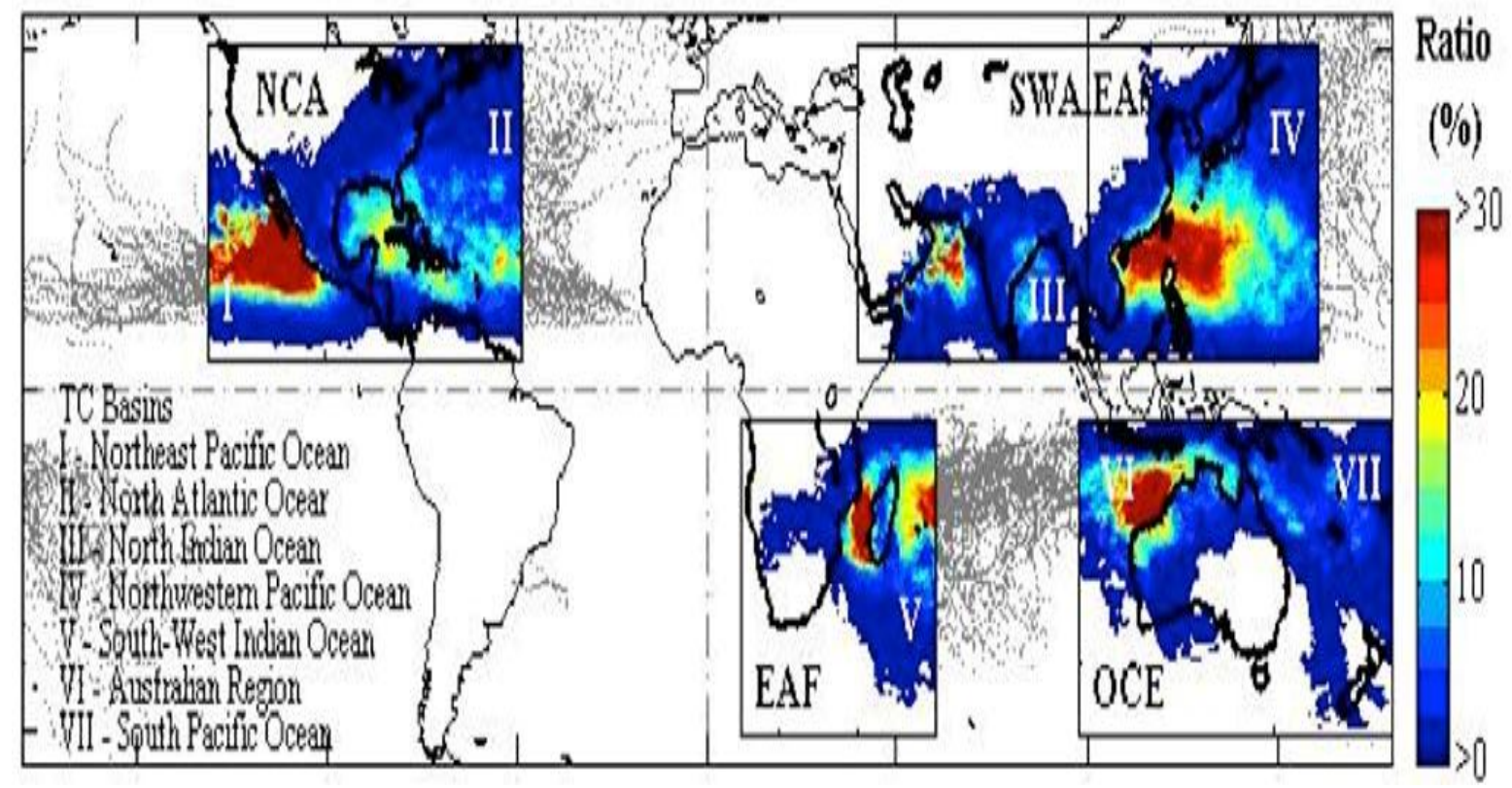

Hình 1. Ảnh hưởng bởi mưa do bão đối với các khu vực trên thế giới [1].

Bảng 1. Tên cơn bão và số trường hợp bão được dùng trong nghiên cứu

\begin{tabular}{lllll}
\hline STT & Tên bão & TH1 & TH2 & TH3 \\
\hline 1 & KETSANA & $00 \mathrm{z} 27 / 09 / 2009$ & $00 \mathrm{z} 28 / 09 / 2009$ & $00 \mathrm{z} 29 / 09 / 2009$ \\
2 & MUJIGAE & $00 \mathrm{z} 09 / 09 / 2009$ & $00 \mathrm{z} 10 / 09 / 2009$ & $00 \mathrm{z} 11 / 09 / 2009$ \\
3 & MIRINAE & $00 \mathrm{z} 30 / 10 / 2009$ & $00 \mathrm{z} 31 / 10 / 2009$ & $00 \mathrm{z} 01 / 11 / 2009$ \\
4 & PARMA & $00 \mathrm{z} 11 / 10 / 2009$ & $00 \mathrm{z} 12 / 10 / 2009$ & $00 \mathrm{z} 13 / 10 / 2009$ \\
5 & CONSON & $00 \mathrm{z} 15 / 07 / 2010$ & $00 \mathrm{z} 16 / 07 / 2010$ & $00 \mathrm{z} 17 / 07 / 2010$ \\
6 & CHANTHU & $00 \mathrm{z} 20 / 07 / 2010$ & $00 \mathrm{z} 21 / 07 / 2010$ & $00 \mathrm{z} 22 / 07 / 2010$ \\
7 & HAIMA & $00 \mathrm{z} 22 / 06 / 2011$ & $00 \mathrm{z} 23 / 06 / 2011$ & $00 \mathrm{z} 24 / 06 / 2011$ \\
8 & NETSAT & $00 \mathrm{z} 27 / 09 / 2011$ & $00 \mathrm{z} 28 / 09 / 2011$ & $00 \mathrm{z} 29 / 09 / 2011$ \\
9 & NALGAE & $00 \mathrm{z} 02 / 10 / 2011$ & $00 \mathrm{z} 03 / 10 / 2011$ & $00 \mathrm{z} 04 / 10 / 2011$ \\
10 & NOCKTEN & $00 \mathrm{z} 28 / 07 / 2011$ & $00 \mathrm{z} 29 / 07 / 2011$ & $00 \mathrm{z} 30 / 07 / 2011$ \\
11 & KAITAK & $00 \mathrm{z} 15 / 08 / 2012$ & $00 \mathrm{z} 16 / 08 / 2012$ & $00 \mathrm{z} 17 / 08 / 2012$ \\
12 & GAEMI & $00 \mathrm{z} 04 / 10 / 2012$ & $00 \mathrm{z} 05 / 10 / 2012$ & $00 \mathrm{z} 06 / 10 / 2012$ \\
13 & SONTINH & $00 \mathrm{z} 26 / 10 / 2012$ & $00 \mathrm{z} 27 / 10 / 2012$ & $00 \mathrm{z} 28 / 10 / 2012$ \\
14 & BEBINCA & $00 \mathrm{z} 21 / 06 / 2013$ & $00 \mathrm{z} 22 / 06 / 2013$ & $00 \mathrm{z} 23 / 06 / 2013$ \\
15 & RUMBIA & $00 \mathrm{z} 29 / 06 / 2013$ & $00 \mathrm{z} 30 / 06 / 2013$ & $00 \mathrm{z} 01 / 07 / 2013$ \\
16 & MANGKHUT & $00 \mathrm{z} 05 / 08 / 2013$ & $00 \mathrm{z} 06 / 08 / 2013$ & $00 \mathrm{z} 07 / 08 / 2013$ \\
17 & NARI & $00 \mathrm{z} 13 / 10 / 2013$ & $00 \mathrm{z} 14 / 10 / 2013$ & $00 \mathrm{z} 15 / 10 / 2013$ \\
18 & WUTIP & $00 \mathrm{z} 28 / 09 / 2013$ & $00 \mathrm{z} 29 / 09 / 2013$ & $00 \mathrm{z} 30 / 09 / 2013$ \\
19 & HAIYAN & $00 \mathrm{z08} / 11 / 2013$ & $00 \mathrm{z} 09 / 11 / 2013$ & $00 \mathrm{z} 10 / 11 / 2013$ \\
\hline
\end{tabular}


Bên cạnh đó, rất nhiều nghiên cứu trong nước và quốc tế đã được thực hiện nhiều nghiên cứu về ảnh hưởng của sự thay đổi độ phân giải, thay đổi các sơ đồ tham số hóa đối lưu đến chất lượng mô phỏng. Các nghiên cứu trước đây đã chỉ ra rằng có độ phân giải cao hơn có thể mô phỏng thực tế hơn hoàn lưu khí quyển quy mô lớn cũng như phân bố lượng mưa toàn cầu và khu vực (Buonomo và ccs, 2007; Gent và ccs, 2010; Jung và ccs, 2012; Kendon và ccs, 2012; Kiều Thị Xin và ccs 2006, Hoàng Đức Cường và ccs, 2008; Trần Tân Tiến và ccs, 2006; Bùi Minh Tăng và ccs, 2011) [3-10]. Như vậy đối với bài toán dự báo mưa nói chung hay dự báo mưa ảnh hưởng do bão nói riêng, việc hạ độ phân giải và thay đổi sơ đồ tham số hóa đối lưu là cần thiết.

\section{Số liệu}

Trong nghiên cứu này thí nghiệm dự báo 19 cơn bão, mỗi cơn bão được dự báo thử nghiệm 3 lần, như vậy số trường hợp dự báo thử nghiệm là 57. Số liệu dự báo GFS của trung tâm NCEP được dùng làm điều kiện ban đầu và điều kiện biên. Số liệu nhiệt độ mặt nước biển trung bình tuần của NOAA được cập nhật cho các trường hợp. Ngoài ra chúng tôi còn sử dụng Số liệu quan trắc được lấy từ hệ thống 176 trạm khí tượng trên phạm vi cả nước.

\section{Phương pháp}

Nghiên cứu sử dụng 2 lưới lồng có độ phân giải lần lượt là $30 \mathrm{~km}$ và $7,5 \mathrm{~km}$. Trong thí nghiệm này nhóm nghiên cứu sử dụng mô hình RAMS (mô hình dự báo khí tượng khu vực do do Đại học Tổng hợp Bang Colorado phát triển) với hai sơ đồ đối lưu là Kuo và Kain-Fritsch (mỗi trường hợpchạy thử nghiệm với 4 phương án khác nhau (Kuo (lưới 1) - Kain-Fritsch (lưới 2) (ký hiệu: K-KF) ; Kuo (lưới 1) - Kuo (lưới 2) (ký hiệu: K-K); Kain-Fritsch (lưới 1) - KainFritsch (lưới 2) (ký hiệu: KF-KF); Kain-Fritsch (lưới 1) - Kuo (lưới 2) (ký hiệu: KF-K)). Hạn dự báo 84 giờ để đánh giá khả năng dự báo lượng mưa do bão bằng mô hình RAMS với hạn dự báo 3 ngày (do số liệu mưa thực đo tính từ $19 \mathrm{~h}$ ). Tâm miền tính tại, $16^{\circ} \mathrm{N}, 108^{\circ} \mathrm{E}$. Ngoài việc hạ độ phân giải lưới và thay đổi các sơ đồ đối lưu, các thông số của mô hình được lựa chọn như sau: sơ đồ bức xạ sóng ngắn của Chen, sóng dài của Mahrer, điều kiện biên xung quanh sơ đồ của Klemp và Wilhelmson.

Kết quả nhận được từ mô hình sẽ được đánh giá với số liệu quan trắc thực, nhằm tìm ra phương án tối ưu để dự báo định lượng mưa.

+ Sai số trung bình ME:

$M E=\frac{1}{n} \sum_{i=1}^{n}\left(F_{\mathrm{i}}-O_{\mathrm{i}}\right)$

+ Sai số trung bình toàn phương RMSE:

RMSE $=\sqrt{\frac{1}{n} \sum_{i=1}^{n}\left(F_{\mathrm{i}}-O_{\mathrm{i}}\right)^{2}}$

Trong đó: $\mathrm{F}_{\mathrm{i}:}$ là số liệu dự báo $\mathrm{O}_{\mathrm{i}:}$ là số liệu quan trắc n: là dung lượng mẫu

Kết quả đánh giá dự báo mưa tại các trạm thông qua các điểm số đánh giá $\mathrm{FBI}, \mathrm{POD}$, $\mathrm{FAR}, \mathrm{CSI}$ và $\mathrm{PC}$ được dựa vào bảng ngẫu nhiên sau (Damrath, 2002) [11]:

Hits $(\mathrm{H})=$ dự báo có + quan trắc có

Misses $(\mathrm{M})=$ dự báo không + quan trắc có

False alarms $(\mathrm{F})=$ dự báo có + quan trắc không

Correct negatives $(\mathrm{CN})=$ dự báo không + quan trắc không

$$
\begin{aligned}
& \mathrm{FBI}=(\mathrm{H}+\mathrm{F}) /(\mathrm{H}+\mathrm{M}) \\
& \mathrm{POD}=\mathrm{H} /(\mathrm{H}+\mathrm{M}) \\
& \mathrm{FAR}=\mathrm{F} /(\mathrm{H}+\mathrm{F}) \\
& \mathrm{CSI}=\mathrm{TS}=\mathrm{H} /(\mathrm{M}+\mathrm{F}+\mathrm{H}) \\
& \mathrm{PC}=(\mathrm{H}+\mathrm{CN}) /(\mathrm{M}+\mathrm{F}+\mathrm{H}+\mathrm{CN})
\end{aligned}
$$

\section{Kết quả}

Tiến hành đánh giá kết quả dự báo mưa tại các trạm do ảnh hưởng của bão đối với 19 cơn bão. Các trạm được sử dụng tùy thuộc vào mỗi cơn bão, các trạm này sẽ được lựa chọn khi trong bán kính $600 \mathrm{~km}$ [12] tính từ tâm thực của bão. Như vậy, số lượng trạm tham gia đánh giá đối với hạn 24,48 và 72 giờ tương ứng là 4645 , 5679 và 5764 trạm. 
Hạn 24 giò

Bảng 2. Điểm số đánh giá mưa hạn 24 giờ (thời hạn dự báo 12-36 giờ)

\begin{tabular}{|c|c|c|c|c|c|c|c|c|c|c|c|c|c|c|c|c|c|}
\hline Ngưỡng & & & $16 \mathrm{~mm}$ & & & & & $>50 \mathrm{~mm}$ & & & & & $00 \mathrm{~mm}$ & & & & \\
\hline $\begin{array}{l}\text { Điểm } \\
\text { số } \\
\text { Phương } \\
\text { án }\end{array}$ & FBI & POD & FAR & CSI & $\mathrm{PC}$ & FBI & POD & FAR & CSI & PC & FBI & POD & FAR & CSI & $\mathrm{PC}$ & ME & RMSE \\
\hline K-K & 1.6 & 0.6 & 0.6 & 0.3 & 0.6 & 0.8 & 0.3 & 0.6 & 0.2 & 0.9 & 0.6 & 0.2 & 0.6 & 0.2 & 1.0 & 4.02 & 32.50 \\
\hline K-KF & 2.7 & 0.9 & 0.7 & 0.3 & 0.5 & 2.0 & 0.4 & 0.8 & 0.1 & 0.8 & 0.7 & 0.1 & 0.8 & 0.1 & 1.0 & 16.46 & 41.20 \\
\hline KF-KF & 2.2 & 0.8 & 0.6 & 0.3 & 0.6 & 1.4 & 0.3 & 0.8 & 0.1 & 0.8 & 0.6 & 0.1 & 0.9 & 0.0 & 1.0 & 9.96 & 39.72 \\
\hline KF-K & 1.1 & 0.5 & 0.6 & 0.3 & 0.7 & 0.6 & 0.2 & 0.6 & 0.2 & 0.9 & 0.5 & 0.2 & 0.7 & 0.1 & 1.0 & 0.06 & 33.43 \\
\hline
\end{tabular}

Độ chính xác $\mathrm{PC}$ của các phương án cũng tương đối đồng đều. Ta thấy PC có xu hướng tăng dần theo các ngưỡng mưa và đều đạt giá trị bằng 1 với ngưỡng mưa $>100 \mathrm{~mm}$. Điều này có thể giải thích bởi công thức tính $\mathrm{PC}$, khi mà ngưỡng mưa càng nhỏ thì giữa khả năng xuất hiện $\mathrm{CN}$ (dự báo không + quan trắc không) càng nhỏ, do đó tỉ lệ giữa tổng của $\mathrm{H}$ và $\mathrm{CN}$ so với tổng $\mathrm{H}+\mathrm{M}+\mathrm{F}+\mathrm{CN}$ càng nhỏ, khi ngưỡng mưa càng tăng tỉ lệ $\mathrm{CN}$ càng lớn, giá trị này chiếm đa số so với các giá trị còn lại nên với không gian mẫu càng lớn thì $\mathrm{PC}$ tiệm cận đến 1 .

Kết quả tính toán sai số cho thấy lượng mưa dự báo luôn lớn hơn lượng mưa quan trắc ở cả 4 phương án đối với hạn dự báo $24 \mathrm{~h}$ (thể hiện ở giá trị $\mathrm{ME}>0$ ), và sai số dự báo mưa nhỏ nhất là của phương án $K-K$ với lượng mưa sai lệch khoảng $32.5 \mathrm{~mm} /$ ngày.

Có thể dễ dàng nhận thấy hai phương án KF-KF và KF-K không phù hợp để dự báo mưa tại hạn dự báo này. Đối với phương án KF-KF, tuy ở ngương mưa 16-50 mm cho khả năng phát hiện hiện tượng tương đối cao tuy nhiên khả năng dự báo diện mưa tương đối kém và đối với các ngưỡng mưa lớn hơn cho thấy độ chính xác và độ tin cậy là thấp nhất so với các phương án khác. Đối với phương án KF-K, tuy giá trị $\mathrm{FBI}$ của phương án này là khá sát với giá trị tối ưu đối với ngưỡng mưa $16-50 \mathrm{~mm}$, nhưng khả năng phát hiện hiện tượng cũng tại ngưỡng mưa này lại thấp nhất so với các phương án khác và với các ngưỡng mưa lớn, chỉ số FBI càng ngày càng thấp. Nhìn chung độ ổn đinh của hai phương án này không cao.
Từ đó có thể thấy rằng đối với hạn dự báo $24 \mathrm{~h}$, có thể sử dụng phương án K-K để đánh giá mưa do bão với ngưỡng dự báo mưa vừa $(16-50 \mathrm{~mm})$ và mưa to $(50-100 \mathrm{~mm})$ còn với ngưỡng dự báo mưa $>100 \mathrm{~mm}$ thì mô hình có độ chính xác không đáng kể. Phương án K-KF tuy cho thấy khả năng phát hiện hiện tượng vượt trội ở ngưỡng dự báo $16-50 \mathrm{~mm}$ và $50-100$ $\mathrm{mm}$ nhưng đến ngưỡng dự báo $>100 \mathrm{~mm}$ lại cho thấy khả năng phát hiện hiện tượng kém hơn so với $\mathrm{K}-\mathrm{K}$, thêm nữa tỷ phần phát hiện sai và $\mathrm{FBI}$ của phương án này cũng lớn hơn so với $\mathrm{K}-\mathrm{K}$ chứng tỏ rằng phương án này có $\mathrm{xu}$ hướng dẫn tới các dự báo khống gây sai số, trong khi K-K cho thấy khả năng dự báo diện mưa ổn định và đồng đều hơn ở các ngưỡng lượng mưa. Cũng cần so sánh về sai số trung bình trong khi cả hai phương án $K-K$ và $K-K F$ dều cho lượng mưa lớn hơn thực tế nhưng giá trị sai số của $\mathrm{K}$ $\mathrm{K}$ nhỏ hơn nhiều so với K-KF.

\section{Hạn 48 giò}

Kết quả đánh giá cho hạn dự báo $48 \mathrm{~h}$ được thể hiện trên Bảng 3 cho thấy xu thế thay đổi các chỉ số theo các ngưỡng mưa khác nhau tương tự như hạn dự báo $24 \mathrm{~h}$. Tuy nhiên cũng cần chú ý một số đặc trưng như:

Chỉ số FBI trong hạn dự báo này của phương án $\mathrm{K}-\mathrm{K}$ khá ổn định trong đó với ngưỡng mưa $>50 \mathrm{~mm}$ chỉ số này đạt giá trị tối uu $(\mathrm{FBI}=1)$.

Phương án $\mathrm{K}-\mathrm{KF}$ và KF-KF vẫn cho thấy tỉ lệ dự báo khống lớn (thể hiện qua các chỉ số FBI và $F A R$ ), tuy nhiên về dự báo lượng mưa theo các ngưỡng mưa thì K-KF lại cho thấy khả năng phát hiện hiện tượng khá cao so với các phương án khác (thể hiện qua chỉ số POD). 
Bảng 3. Điểm số đánh giá mưa hạn 48 giờ (thời hạn dự báo 36-60 giờ)

\begin{tabular}{|c|c|c|c|c|c|c|c|c|c|c|c|c|c|c|c|c|c|}
\hline Ngưỡng & & & $16 \mathrm{~mm}$ & & & & & $50 \mathrm{~mm}$ & & & & & $100 \mathrm{~m}$ & & & & \\
\hline $\begin{array}{l}\text { Điểm } \\
\text { số } \\
\text { Phương } \\
\text { án } \\
\end{array}$ & FBI & POD & FAR & CSI & $\mathrm{PC}$ & FBI & POD & FAR & CSI & PC & FBI & POD & FAR & CSI & PC & ME & RMSE \\
\hline K-K & 1.3 & 0.4 & 0.7 & 0.2 & 0.6 & 1.0 & 0.2 & 0.8 & 0.1 & 0.9 & 0.8 & 0.2 & 0.8 & 0.1 & 1.0 & 3.00 & 37.41 \\
\hline K-KF & 2.8 & 0.8 & 0.7 & 0.3 & 0.4 & 2.4 & 0.3 & 0.9 & 0.1 & 0.8 & 0.5 & 0.1 & 0.8 & 0.1 & 1.0 & 16.04 & 40.76 \\
\hline KF-KF & 2.3 & 0.7 & 0.7 & 0.3 & 0.5 & 1.5 & 0.2 & 0.9 & 0.1 & 0.8 & 0.4 & 0.0 & 0.9 & 0.0 & 1.0 & 9.18 & 38.53 \\
\hline KF-K & 0.7 & 0.3 & 0.6 & 0.2 & 0.7 & 0.3 & 0.1 & 0.8 & 0.0 & 0.9 & 0.2 & 0.0 & 0.9 & 0.0 & 1.0 & -5.38 & 34.16 \\
\hline
\end{tabular}

Xét về sai số thì phương án KF-K cho lượng mưa dự báo nhỏ hơn mưa quan trắc $(\mathrm{ME}<0)$ trái ngược với các phương án khác, tuy nhiên phương án này lại cho lượng mưa sai lệch so với quan trắc là nhỏ nhất $(34.16 \mathrm{~mm} / \mathrm{ngày})$.

Tương tự như hạn dự báo $24 \mathrm{~h}$ có thể loại bỏ hai phương án KF-KF và KF-K trong hạn dự báo này, do hai phương án này cho thấy khả năng phát hiện hiện tượng không cao, dự báo khống lớn và điểm số thành công thấp.

Trong hạn dự báo này, với ngưỡng mưa 16$50 \mathrm{~mm}$ có thể chọn phương án K-KF để dự báo lượng mưa do khả năng phát hiện hiện tượng của phương án này lớn hơn nhiều so với các phương án khác. Với ngưỡng lượng mưa lớn 50-100 có thể chọn phương án K-K để dự báo mưa do phương án này cho kết quả diện mưa dự báo trùng với diện mưa quan trắc đồng thời khả năng phát hiện hiện tượng cũng như tỷ phần phát hiện sai tương đương với các phương án khác. Với ngưỡng mưa lớn hơn $100 \mathrm{~mm}$ mô hình cho kết quả dự báo không chính xác.

\section{Hạn 72 giò}

Hạn dự báo 72 giờ, xu thế biến đổi các chỉ số theo ngưỡng mưa cũng vẫn tương tự như hạn dự báo $48 \mathrm{~h}$ và $24 \mathrm{~h}$.

Phương án KF-K vẫn cho lượng mưa dự báo nhỏ hơn lượng mưa quan trắc và cho giá trị sai số nhỏ hơn các phương án khác (RMSE $=28.78 \mathrm{~mm} /$ ngày)

Với hạn dự báo này ta có thể thấy hai phương án $K F-K$ và $K-K$ dự báo lượng mưa kém. Tuy $K F-K$ và $K-K$ cho kết quả sai số lượng mưa khá nhỏ nhưng các chỉ số đều cho thấy hai phương án này không bắt được hiện tượng và chủ yếu là dự báo sót, cũng có thể do nguyên nhân này nên sai số của phương án mới cho giá trị nhỏ hơn các phương án khác.

Phương án KF-KF tới thời hạn dự báo này có vẻ chất lượng dự báo được cải thiện so với hai thời hạn dự báo trước, thể hiện ở chỉ số POD tại ngưỡng mưa $16-50 \mathrm{~mm}$ cao hơn hẳn so với hai phương án $K-K$ và $K F-K$ và $F B I=1.1$ tại ngưỡng mưa $>100 \mathrm{~mm}$ tuy nhiên xét về độ ổn định của các chỉ số thì với thời hạn này nên chọn phương án K-KF để dự báo mưa với ngưỡng mưa vừa và mưa to còn ngưỡng mưa $>100 \mathrm{~mm}$ mô hình vẫn thể hiện độ chính xác không đáng kể.

So sánh các chỉ số giữa các hạn dự báo với nhau ta thấy:

Hạn dự báo càng dài thì hai phương án $\mathrm{K}$ KF và KF-KF cho dự báo khống càng lớn. Còn hai trường hợp $\mathrm{K}-\mathrm{K}$ và $\mathrm{KF}-\mathrm{K}$ cho dự báo sót càng nhiều.

Phương án KF-KF cho tî̉ lệ dự báo khống lớn, khả năng phát hiện hiện tượng không lớn (chỉ phát hiện tốt ở ngưỡng mưa 16-50 mm ), độ tin cậy không cao.

Phương án KF-K cho sai số lượng mưa dự báo so với thực tế là nhỏ nhất, tuy nhiên qua việc xem xét tính ổn đinh, xu thế biến đổi các chỉ số thì phương án này cho kết quả độ tin cậy không cao.

Phương án K-KF cho thấy khả năng phát hiện hiện tượng vượt trội so với các phương án còn lại tuy nhiên dự báo khống cũng lớn nhất trong các phương án ( kể cả về diện và lượng), chỉ phù hợp với các dự báo hạn dài với ngưỡng dự báo mưa tối đa là mưa to. 
Bảng 4. Điểm số đánh giá mưa hạn 72 giờ (thời hạn dự báo 60-84 giờ)

\begin{tabular}{|c|c|c|c|c|c|c|c|c|c|c|c|c|c|c|c|c|c|}
\hline \multirow{2}{*}{$\begin{array}{l}\text { Ngưỡng } \\
\text { Điểm số } \\
\text { Phương án }\end{array}$} & \multicolumn{5}{|c|}{$>16 \mathrm{~mm}$} & \multicolumn{5}{|c|}{$>50 \mathrm{~mm}$} & \multicolumn{5}{|c|}{$>100 \mathrm{~mm}$} & \multirow[b]{2}{*}{ ME } & \multirow[b]{2}{*}{ RMSE } \\
\hline & FBI & POD & FAR & CSI & $\mathrm{PC}$ & FBI & POD & FAR & CSI & $\mathrm{PC}$ & FBI & POD & FAR & CSI & $\mathrm{PC}$ & & \\
\hline K-K & 1.4 & 0.3 & 0.8 & 0.1 & 0.6 & 0.8 & 0.1 & 0.9 & 0.0 & 0.9 & 0.3 & 0.0 & 0.9 & 0.0 & 1.0 & 1.83 & 31.41 \\
\hline $\mathrm{K}-\mathrm{KF}$ & 3.8 & 0.8 & 0.8 & 0.2 & 0.4 & 3.6 & 0.3 & 0.9 & 0.1 & 0.8 & 0.5 & 0.1 & 0.8 & 0.1 & 1.0 & 20.60 & 39.83 \\
\hline KF-KF & 2.9 & 0.6 & 0.8 & 0.2 & 0.5 & 2.3 & 0.1 & 0.9 & 0.0 & 0.8 & 1.1 & 0.0 & 1.0 & 0.0 & 1.0 & 13.70 & 40.12 \\
\hline KF-K & 0.5 & 0.1 & 0.8 & 0.1 & 0.8 & 0.2 & 0.1 & 0.7 & 0.0 & 0.9 & 0.1 & 0.0 & 0.9 & 0.0 & 1.0 & -5.13 & 28.78 \\
\hline
\end{tabular}

Phương án K-K, qua tính toán so sánh các chỉ số và các sai số cho thấy phương án này dự báo lượng mưa và diện mưa tương đối tốt đối với hạn dự báo $48 \mathrm{~h}$ trở xuống.

Với ngưỡng mưa $>100 \mathrm{~mm}$ tất cả các phương án đều cho thấy mô hình RAMS không có khả năng dự báo lượng mưa tại ngưỡng mưa này.

\section{Kết luận}

Thông qua việc tính toán, thống kê số liệu dự báo lượng mưa của các phương án có thể đưa đến một số kết luận: Hai phương án KF-KF , KF-K cho kết quả dự báo lượng mưa kém không thể đưa vào dự báo mưa do bão; Với hạn dự báo $24 \mathrm{~h}$ nên sử dụng phương án K-K để dự báo ngưỡng mưa vừa $(16-50 \mathrm{~mm})$ và mưa to (50-100mm); với hạn dự báo 48 h nên sử dụng phương án K-KF để dự báo mưa do bão cho ngưỡng mưa vừa và phương án $\mathrm{K}-\mathrm{K}$ để dự báo cho ngưỡng mưa to; với hạn dự báo $72 \mathrm{~h}$ nên sử dụng phương án $\mathrm{K}-\mathrm{KF}$ đề dự báo ngưỡng mưa vừa và mưa to. Với ngưỡng mưa trên $100 \mathrm{~mm}$ đối với tất cả các hạn dự báo, độ chính các của mô hình RAMS rất kém, hầu như không dự báo được. Từ những kết quả đã đạt được nhóm nghiêm cứu hi vọng sẽ đóng góp thêm những thông tin hữu ích góp phần vào việc tăng độ chính xác của dự báo mưa do bão trên khu vực Việt Nam trong thời gian tới.

\section{Tài liệu tham khảo}

[1] Prat, O.P., and B.R. Nelson, 2013. Mapping the world's tropical cyclone rainfall contribution over land using TRMM Multisatellite Precipitation
Analysis. Water Resources Research. DOI: 10.1002/wrcr.20527

[2] Chen L. et al. (2010), An Overview of Research and Forecasting on Rainfall Associated with Landfalling Tropical Cyclones, ADVANCES IN ATMOSPHERIC SCIENCES, VOL. 27, NO. 5, 2010, 967.

[3] Buonomo, E., R. Jones, C. Huntingford, and J. Hannaford (2007), On the robustness of changes in extreme precipitation over Europe from two high resolution climate change simulations, Q. J. R. Meteorol. Soc., 133(622), 65-81.

[4] Gent, P. R., S. G. Yeager, R. B. Neale, S. Levis, and D. A. Bailey (2010), Improvements in a half degree atmosphere/land version of the CCSM, Clim. Dyn., 34(6), 819-833.

[5] Jung, T., et al. (2012), High-resolution global climate simulations with the ECMWF model in Project Athena: Experimental design, model climate, and seasonal forecast skill, J. Clim., 25(9), 3155-3172.

[6] Kendon, E. J., N. M. Roberts, C. A. Senior, and M. J. Roberts (2012), Realism of rainfall in a very high-resolution regional climate model, J. Clim., 25(17), 5791-5806.

[7] Kiều Thị Xin, Đề tài độc lập cấp nhà nước (20042006): Nghiên cứu dự báo mưa lớn diện rộng bằng công nghệ hiện đại phục vụ phòng cống lũ lụt ở Việt Nam.

[8] Hoàng Đức Cường, Đề tài cấp bộ TNMT 2008 "Nghiên cứu thử nghiệm dự báo mưa lớn ở Việt Nam bằng mô hình MM5"

[9] Trần Tân Tiến, Đề tài cấp ĐHQGHN 2004, Xây dựng công nghệ dự báo lũ bằng mô hình số thời hạn 3 ngày cho khu vực Trung Bộ Việt Nam. QG2004-2006

[10] Bùi Minh Tăng Đề tài độc lập cấp nhà nước 2014 "Nghiên cứu xây dựng công nghệ dự báo mưa lớn thời hạn 2-3 ngày phục vụ công tác cảnh báo sớm lũ lụt khu vực miền trung Việt Nam" 
[11] Damrath, U., (2004) "Verification against precipitation observations of a high density network - what did we learn” Intl. Verification Methods Workshop, 15-17 September 2004, Montreal, Canada. [Available online at 82 http://www.bom.gov.au/bmrc/wefor/staff/eee/veri f/Workshop.
[12] 2004/presentations/5.3 Damrath.pdf]; November 2006.

[13] Gleason, B., 2006: Characteristics of tropical cyclone rainfall in the United States. 27th Conference on Hurricanes and Tropical Meteorology, Session 16C, Tropical Cyclones and Climate V-Atlantic Basin.

\title{
Assessing Prediction of Tropical Cyclone Rainfall by RAMS Model
}

\author{
Cong Thanh ${ }^{1}$, Tran Tien Dat ${ }^{2}$, Vu Thanh Hang ${ }^{1}$ \\ ${ }^{1}$ Meteorology hydrology and oceanography of Faculty, VNU University of Science, \\ 334 Nguyen Trai, Thanh Xuan, Hanoi \\ ${ }^{2}$ Technology Application and Training Center for Hydro-meteorology and Enviroment
}

\begin{abstract}
This study assesses the predictability rain storm of the RAMS model with 3 day term for Viet Nam region, use two convective schemer: Kuo and Kain-fritsch. The researchers plan to conduct experiments with nested grid method and change two convection to grid 1 and grid 2, which resulted in: The $24 \mathrm{~h}$ term forecasts, we should use K-K plan to forecast medium rain $(16-50 \mathrm{~mm})$ and heavy rain $(50-100 \mathrm{~mm})$; term forecast for $48 \mathrm{~h}$ we should use K-KF plan to forecast medium rain threshold and K-K plan to forecast heavy rain threshold; with $72 \mathrm{~h}$-term forecasts we should use K-KF plan to forecast medium rain and heavy rain threshold. With more than $100 \mathrm{~mm}$ rainfall thresholds for all term forecasts, the accuracy of the model RAMS was poor, hardly predictable. The results of this study can give forecast more predict information to serve the forecasted rain storm in the area of Vietnam.
\end{abstract}

Keywords: Research and Forecasting on Rainfall, Tropical Cyclones. 